\title{
Uma introdução aos métodos mistos
}

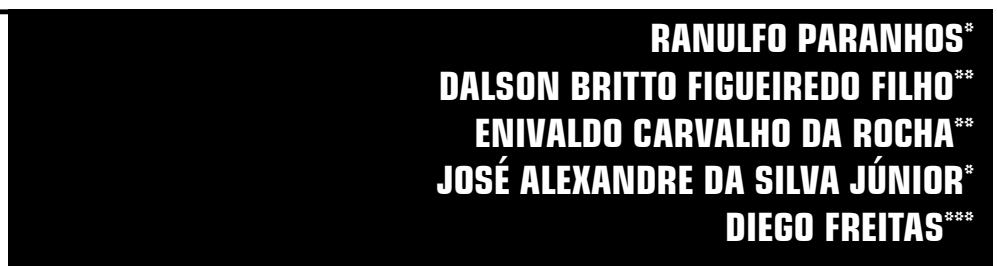

Resumo

Quais são as vantagens da triangulação metodológica? Apesar de ser consensual a importância da combinação de técnicas quantitativas e qualitativas, ainda são raros os trabalhos que efetivamente utilizam uma abordagem multimétodo. Este artigo apresenta uma introdução aos métodos mistos. Nosso público alvo são estudantes de graduação e pós-graduação em estágios iniciais de treinamento. Metodologicamente, sintetizamos as principais recomendações da literatura e utilizamos dois exemplos para ilustrar como a combinação de técnicas pode ser empregada em desenhos de pesquisa empíricos. Com este artigo, esperamos difundir a utilização de métodos mistos nas Ciências Sociais brasileiras ${ }^{1}$.

Palavras-chaves: Métodos Mistos. Triangulação Metodológica. Ciência Política.

\footnotetext{
* Universidade Federal de Alagoas (Brasil), ${ }^{* *}$ Universidade Federal de Pernambuco (Brasil),

${ }^{* * *}$ Centro Universitário Tiradentes (Brasil).

${ }^{1}$ Este trabalho se beneficiou dos comentários dos membros do Grupo de Métodos de Pesquisa da Universidade Federal de Pernambuco (UFPE) e Grupo de Pesquisa Cidadania e Políticas Públicas (UFAL), além disso agradecemos ao CNPQ e a FACEPE pelo apoio financeiro.
} 


\title{
An Introduction to mixed methods
}

\begin{abstract}
What are the advantages of methodological triangulation? Although there is a general consent about the importance of mixing quantitative and qualitative methods, studies that effectively use multi method approach are still rare. This article provides an introduction to mixed methods. Our target audience is both undergraduate and graduate students in early stages of training. Methodologically, we will summarize the main recommendations in the literature and use two examples to show how mixed methods can be applied to empirical research designs. With this paper we hope to diffuse the utilization of mixed methods in Brazilian Social Sciences.

Keywords: Mixed Methods. Methodological Triangulation. Political Science.
\end{abstract}

Mixed methods research has become popular as the newest development in research methods and in approaches to mixing quantitative and qualitative research.

John Creswell

Together we stand, divided we fall

Pink Floyd 


\section{Introdução}

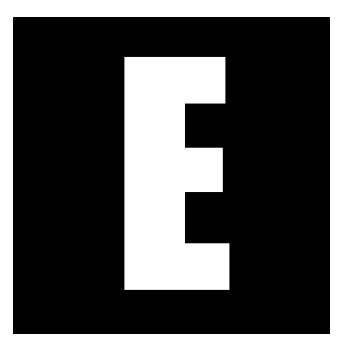

m 1970, Denzin (1970) afirmou que a combinação de diferentes teorias, métodos e fontes de dados pode ajudar a superar o viés natural que atinge estudos com abordagens singulares (single-method, single-observer, single-theory studies) (Denzin, 1970). Depois de mais de 30 anos desse alerta, a combinação de métodos permanece uma prática analiticamente desejável, mas raramente utilizada (Niglas, 2004; Bryman; Bell, 2006; Wooley, 2008). Como explicar esse paradoxo? Acreditamos que o principal obstáculo à abordagem multimétodo está mais relacionado à falta de treinamento específico, do que a uma opção ontológica e/ ou epistemológica da comunidade científica².

Em geral, o pesquisador experiente em conduzir entrevistas desconhece pressupostos básicos de um modelo de regressão. Similarmente, pesquisadores com avançado conhecimento de Econometria não sabem como realizar uma observação participante. A combinação de métodos depende de uma reestruturação dos currículos de graduação e pós-graduação no sentido de assegurar não só a oferta regular e intensiva de cursos de métodos quantitativos e qualitativos, mas principalmente uma disciplina específica sobre como integrá-los ${ }^{3}$. Além dos benefícios analíticos da triangulação, tem-se o ganho técnico para o próprio pesquisador ${ }^{4}$.

\footnotetext{
${ }^{2}$ Para Sandelowski (2000), "researchers have turned to mixed-method techniques to expand the scope and improve the analytic power of their studies. Yet there is still relatively little direction on and much confusion about how to combine qualitative and quantitative techniques" (Sandelowski, 2000: 246).

${ }^{3}$ Creswell (2009) aponta "note the challenges this form of research poses for the inquirer. These include the need for extensive data collection, the time-intensive nature of analyzing both text and numeric data, and the requirement for the researcher to be familiar with both quantitative and qualitative forms of research" (Creswell, 2009: 881).

${ }^{4}$ Para Marques et al. (2007), "a produção científica brasileira, em geral, e o ensino de ciências sociais, em particular, apresentam uma lacuna significativa no campo das técnicas de pesquisa,
} 
Além disso, como são poucos os trabalhos que de fato integram diferentes técnicas, os interessados em métodos mistos também sofrem com a ausência de exemplos. Ainda, a literatura pedagógica em português é limitada, o que dificulta sobremaneira a efetiva integração de técnicas quantitativas e qualitativas (Woolley, 2008). Para Tarrow (1995), essa é uma das limitações de Design Social Inquiry (King; Keohane; Verba, 1994)

Este artigo apresenta uma introdução aos métodos mistos. $\mathrm{O}$ foco repousa sobre a definição do conceito, a descrição das características e a identificação das situações em que a combinação de técnicas é analiticamente desejável. O desenho de pesquisa sumariza as principais recomendações da literatura e utiliza dois exemplos para ilustrar como a triangulação de técnicas pode ser empregada. Com este artigo, esperamos difundir a utilização de métodos mistos na Ciência Política brasileira.

O restante do artigo está dividido da seguinte forma: a próxima seção discute a ontologia e a epistemologia subjacente aos métodos quantitativos e qualitativos. A terceira seção apresenta a definição, a classificação e as características dos métodos mistos. Na sequência, apresentamos dois exemplos sobre como técnicas quantitativas e qualitativas podem ser integradas. A última seção sumariza as conclusões.

\section{Métodos quantitativos versus métodos qualitativos?}

A tensão entre métodos quantitativos e qualitativos remonta um debate ontológico e epistemológico no âmbito da Filosofia da Ciência (Bry-

sejam elas quantitativas ou qualitativas, sejam exploratórias ou analíticas. O problema é causado em parte por um círculo vicioso, visto que poucos entre nós foram formados (quando estudantes) para ensinar esse assunto, o que mantém as novas gerações de cientistas sociais pouco conhecedoras das principais ferramentas técnicas existentes" (Marques et al., 2007: 142).

${ }^{5}$ Para Tarrow (1995), "while they offer a good deal of generous (at times patronizing) advice to qualitatively oriented scholars, they say very little about how qualitative approaches can be combined with quantitative research" (Tarrow, 1995: 471). 
man, 1984; Morgan, 2007). De um lado, tem-se a visão positivista de que os métodos e técnicas das Ciências Humanas devem aproximar-se daqueles das Ciências Naturais ${ }^{6}$. De outro, os seguidores do interpretativismo${ }^{7}$ defendem que as Ciências Naturais e Sociais constituem abordagens distintas e, por isso, requerem métodos específicos. Estudar um componente químico em um laboratório é totalmente diferente da investigação dos fenômenos sociais. Para a posição interpretativista, existe uma diferença fundamental entre os mundos natural e social: o significado. Por isso, a realidade social deve ser interpretada. Na tradição sociológica alemã, o termo verstehen é utilizado como sinônimo de compreensão/interpretação. Dessa forma, o principal componente da abordagem hermenêutica é o significado subjetivo das ações (Marsh; Furlong, 2002: 24) ${ }^{8}$.

Antes de apresentar a definição e as potencialidades da abordagem multimétodo, é importante revisar a lógica subjacente aos métodos quantitativos e qualitativos. Para Creswell (2012), os dados quantitativos, como números e indicadores, podem ser analisados com auxílio da Estatística (frequência, média, mediana, moda, etc.) e revelar informações úteis, rápidas

\footnotetext{
${ }^{6}$ Della Porta e Keating (2008) argumentam que para a concepção positivista "o mundo existe de forma objetiva, de forma independente do pesquisador, sendo possível conhecer integralmente a realidade. O objetivo do pesquisador é descrever e analisar essa realidade (...) assim como nas ciências naturais, existem regras sistemáticas e regularidades associadas ao objeto de estudo" (Della Porta; Keating, 2008: 23).

${ }^{7}$ Brady e Collier (2004) definem construtivismo como "a research tradition focused on how social and psychological processes influence the way people view, and in part, create reality. It is the study of how human beings, individually and collectively, constitute their world" (Brady; Collier, 2004: 280).

${ }^{8}$ Os relativistas se aproximam daquilo que Tilly (2001) denominou de visão cética. Para os céticos, os fenômenos políticos são considerados demasiadamente complexos e contingentes. $\mathrm{O}$ pesquisador pode tentar reconstruir as experiências dos atores, descrever, interpretar e conferir significado subjetivo às ações, mas não existe preocupação em desenvolver explicações sistemáticas para compreender fenômenos sociais e políticos. A conclusão é bastante direta: a busca por explicações causais é estéril. Ao invés disso, as Ciências Sociais devem se ocupar com a interpretação das ações dos atores sociais, buscando revelar os seus significados subjetivos.
} 
e confiáveis a respeito de um grande número de observações. Segundo o autor, as técnicas qualitativas, como entrevistas abertas, fornecem informações sobre a própria fala dos entrevistados, oferecendo diferentes perspectivas sobre o tema e delineando os aspectos subjetivos do fenômeno.

Entendemos que tanto as técnicas quantitativas quanto as qualitativas têm potencialidades e limitações. Em geral, elas são utilizadas com propósitos distintos. A vantagem da integração consiste em retirar o meIhor de cada uma para responder uma questão específica. A figura 1 ilustra esse argumento.

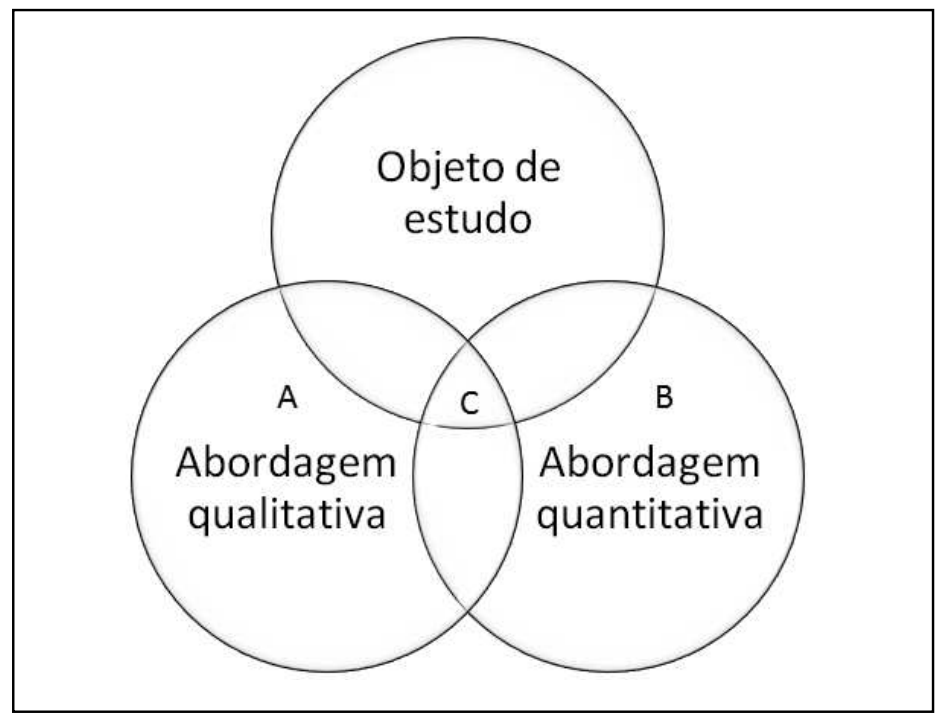

Figura 1. Complementariedade das abordagens

Fonte: elaboração dos autores a partir de Gorard e Taylor (2004)

O objetivo da ciência é descrever/interpretar/explicar/predizer a realidade. Cada abordagem tem a sua contribuição específica (A ou B). A integração permite que uma área inexplorada (C) seja incorporada ao modelo analítico, favorecendo a construção de um desenho de pesquisa mais robusto. Small (2011) identifica duas principais abordagens de integração de métodos. Uma que combina dados quantitativos e qualitativos 
(mixed data-collection studies) e outra que combina técnicas quantitativas e qualitativas (mixed data-analysis studies). A literatura sugere dois principais argumentos para justificar a importância da integração, seja de dados, seja de técnicas: (1) confirmação e (2) complementariedade.

$\mathrm{Na}$ perspectiva confirmatória, quanto mais convergentes forem os resultados observados utilizando diferentes tipos de dados e/ou técnicas, mais consistentes são os resultados da pesquisa. Uma das principais funções da triangulação é garantir que os resultados não dependam da natureza dos dados e/ou das técnicas utilizadas. Por exemplo, suponha que em uma pesquisa sobre um determinado tema, o pesquisador combine técnicas de survey, com entrevistas em profundidade, análise documental e grupos focais. Quanto mais similares forem as inferências, maior é a consistência dos seus resultados. Sempre que o pesquisador identificar a existência de um puzzle na literatura associado a uma divergência na formatação dos desenhos de pesquisa e/ou tipos de dados, tem-se uma importante motivação para adotar uma abordagem multimétodo.

Por sua vez, na perspectiva de complementariedade, o objetivo é ponderar as vantagens e limitações de cada técnica específica e/ou tipo de dado. Por exemplo, ao analisar uma grande base de dados, não é possível identificar os valores/sentimentos dos respondentes. Cada tipo de dado e/ou técnica de pesquisa vai contribuir com uma parcela específica de conhecimento a respeito de um determinado objeto de estudo. A vantagem fundamental da integração é maximizar a quantidade de informações incorporadas ao desenho de pesquisa, favorecendo o seu aprimoramento e elevando a qualidade das conclusões do trabalho'.

\footnotetext{
${ }^{9}$ De acordo com Small (2011), durante a década de 2000, a maior parte dos desenhos de pesquisa que combinaram diferentes tipos de dados utilizaram a perspectiva de complementariedade em detrimento da abordagem confirmatória. Para ele, "most of these studies have employed one of the two approaches: (a) using either textual or small-samples (qualitative) data to interpret the results derived from large-sample (quantitative) data and (b) using large-sample (quantitative) data to test results derived from small-sample (qualitative) data" (Small, 2011: 65).
} 


\section{O que são métodos mistos e como podem ser utilizados? ${ }^{10}$}

Creswell e Plano Clark (2011) definem métodos mistos como um procedimento de coleta, análise e combinação de técnicas quantitativas e qualitativas em um mesmo desenho de pesquisa. O pressuposto central que justifica a abordagem multimétodo é o de que a interação entre eles fornece melhores possibilidades analíticas. Neste trabalho adotamos a definição proposta por Johnson e Onwuebuzie (2004) de que métodos mistos são "the class of research where the researcher mixes or combines quantitative and qualitative research techniques, methods, approaches, concepts or language into a single study" (Johnson; Onwuebuzie, 2004: 17).

Depois de definir o conceito, o próximo passo é identificar as situações em que o pesquisador deve combinar métodos quantitativos e qualitativos. Toda investigação científica começa com o estabelecimento de uma questão de pesquisa. Por exemplo, Dahl (2012) questionou: "Dado um regime em que os opositores do governo não possam se organizar aberta e legalmente em partidos políticos para fazer-lhe oposição em eleições livres e idôneas, que condições favorecem ou impedem sua transformação num regime do qual isto seja possível?" A lógica subjacente da integração é oferecer uma resposta mais robusta à questão proposta, do

\footnotetext{
${ }^{10}$ As leituras obrigatórias sobre métodos mistos são Creswell $(2009 ; 2012)$ e Tashakkori e Teddlie (2003). Para um trabalho sobre a definição conceitual de combinação de métodos ver Johnson, Onwuegbuzie e Turner (2007) e Johnson e Onwuegbuzie (2004). Castro et al. (2010) apresentam diferentes formas de combinação de métodos. Para uma discussão detalhada sobre a evolução histórica da combinação entre métodos quantitativos e qualitativos ver Creswell e Plano Clark (2011) e Tashakkori e Teddlie (1998). Para trabalhos clássicos ver Caracelli e Greene (1997). Para uma introdução ao tema ver Small (2011). Tarrow (1995), Yin (2006) e Woolley (2008) discutem os desafios à integração de métodos quantitativos e qualitativos. Jick (1979) discute o conceito de triangulação de métodos. Para uma discussão sobre a complementariedade dos métodos em português ver Ruiz (2004). Especificamente em Ciência Política ver o artigo de Lieberman (2005) sobre nested analysis. Exemplos de periódicos especializados na combinação de métodos são o Journal of Mixed Methods Research e o International Journal of Multiple Research Approaches.
} 
que aquela que seria produzida por um desenho de pesquisa ou somente quantitativo ou somente qualitativo (Yin, 2006). Para que isso seja efetivamente realizado é necessário que o pesquisador pondere a combinação de métodos durante as seguintes etapas:

(1) questão de pesquisa;

(2) unidade de análise;

(3) amostra;

(4) coleta de dados

(5) estratégias de análise.

No que diz respeito à primeira etapa, deve-se assegurar uma única questão de pesquisa que possa ser investigada por diferentes técnicas ou duas ou mais questões de pesquisa complementares que possam ser investigadas por diferentes técnicas. Por exemplo, é possível inquirir quais são as variáveis que explicam a mobilidade social. Inicialmente o pesquisador pode utilizar um modelo de regressão para estimar o impacto da escolaridade sobre a renda individual. Em um segundo momento, ele pode utilizar entrevistas estruturadas e grupo focal com a mesma amostra ou com outro grupo para tentar compreender como variam opiniões/sentimentos/valores em relação aos fatores que explicam a ascensão social.

Em relação à unidade de análise ${ }^{11}$, é importante definir a unidade básica de análise do desenho de pesquisa. Depois disso, o desafio é garantir a sua homogeneidade contra a tendência natural de que diferentes técnicas privilegiam unidades distintas. Por exemplo, em um estudo sobre o impacto da independência do ministério público sobre a corrupção, a unidade de análise é a própria instituição. É possível então coletar dados referentes ao desenho institucional de cada caso e depois analisar descri-

\footnotetext{
${ }^{11}$ Definimos unidade de análise como os casos da pesquisa, as linhas da planilha. A unidade de análise pode ser indivíduos, grupos, municípios, estados, países, etc.
} 
tivamente e/ou testar hipóteses. Em um segundo momento, o pesquisador pode entrevistar os membros da instituição. Tem-se, aqui, uma nova unidade de análise.

A amostra é um elemento central na pesquisa científica. É a partir dela que os pesquisadores coletam informações sobre diferentes objetos de estudo, selecionam as variáveis analiticamente relevantes e conduzem o próprio desenho de pesquisa. Existem duas principais razões para utilizar amostras: (1) economia de tempo e (2) economia de recursos ${ }^{12}$. Em geral, catalogar informações sobre todas as observações do universo (censo) pode inviabilizar alguns desenhos de pesquisa. Por exemplo, suponha que uma pesquisa tem como objetivo examinar a percepção de sindicalizados sobre a atuação de seus dirigentes. Logisticamente, não faz sentido nenhum entrevistar todos os filiados. A pesquisa seria demasiadamente onerosa e demorada. Além disso, o esforço computacional necessário para trabalhar com amostras é bem menor do que aquele empregado para analisar grandes bases de dados. Dessa forma, sempre que as amostras forem corretamente selecionadas, as inferências produzidas serão precisas, confiáveis e detalhadas ${ }^{13}$.

Quando o objetivo é realizar generalizações válidas para a população, deve-se assegurar a aleatoriedade da amostra no intuito de garantir a equiprobabilidade, ou seja, todos os casos têm a mesma chance de serem selecionados. No entanto, muitas vezes o pesquisador está interessado em realizar um estudo de caso e/ou comparação de poucos casos, de

\footnotetext{
${ }^{12}$ A depender do tamanho da população, o censo pode demorar tanto que a própria população irá se modificar, de modo que ao final da coleta, os dados já não descrevem acuradamente toda a população. Além disso, os recursos computacionais necessários para trabalhar com amostras são mais reduzidos, o que facilita a análise de dados. Em síntese, recursos, tempo e precisão: esses são os principais argumentos em favor da utilização de amostras em detrimento da população em qualquer tipo de pesquisa.

${ }_{13}$ Para mais informações sobre técnicas de amostragem ver Pfeffermann e Rao (2009). Para uma abordagem mais introdutória, ver Kalton (1983).
} 
forma que a seleção não é aleatória, mas sim guiada por critérios teóricos e/ou empíricos. Na perspectiva de integração de técnicas, uma das possibilidades analíticas de seleção da amostra é garantir que ela seja aninhada (nested). Por exemplo, em um estudo sobre desempenho escolar, o pesquisador seleciona escolas dentro de um estado/cidade, alunos de diferentes séries e turmas. No primeiro momento ele pode utilizar variáveis referentes à escola (orçamento, formação do quadro de professores, estrutura física, etc.). Posteriormente, ele pode coletar informações a respeito dos próprios alunos, seja através de testes padronizados de desempenho, seja através de entrevistas e/ou grupo focal.

Cada método de pesquisa utiliza diferentes formas de coletar os dados. Os entrevistadores utilizarão algum aparelho eletrônico para registrar o áudio da conversa, além de anotações. Em uma pesquisa de survey, o questionário é o item obrigatório para coletar as informações de interesse. Um desenho de pesquisa multimétodo não pode ser diferente. Na verdade, é desejável que itens/temas/assuntos sejam compartilhados entre diferentes técnicas. Por exemplo, incluir em um questionário um item que sumarize a ideia, ainda que de forma geral, do roteiro da entrevista. Para Yin (2006), quanto maior o nível de sobreposição e/ou complementariedade dos itens, maior será o nível de integração das diferentes técnicas de pesquisa.

Por fim, em relação às estratégias de análise, o pesquisador deve maximizar a harmonia entre as lógicas quantitativas e qualitativas. Por exemplo, ao utilizar um modelo de regressão multivariada para estimar o valor da variável dependente, o pesquisador está interessado em identificar o impacto de cada fator sobre a variável resposta. Uma forma de integrar a estratégia de análise é realizar um estudo de caso com o mesmo objetivo. Para aquele determinado caso, quais são as variáveis que explicam a variável dependente? Geralmente essas estratégias não são integradas e acabam sendo utilizadas de forma independente. Recomendamos que os 
pesquisadores dediquem muito tempo, talento e energia na elaboração da estratégia analítica de seus desenhos de pesquisa, sob pena de perder o que há de mais benéfico na integração de métodos.

\subsection{Métodos mistos: principais características}

Small (2011) classifica os estudos de acordo com a sequência em que os diferentes tipos de dados são coletados. Na perspectiva sequencial, existe uma assimetria temporal entre a coleta de dados quantitativos e qualitativos. Um caminho promissor é realizar um estudo de caso após examinar os resultados de uma análise multivariada, buscando melhor compreender uma observação destoante. Outra possibilidade é entrevistar os mesmos indivíduos em períodos temporais distintos, realizar uma comparação das respostas (antes/depois) e selecionar os cinco por cento superiores e inferiores para entrevistas em profundidade. $\mathrm{Na}$ abordagem concorrente, os dados são coletados de forma simultânea. Isso pode acontecer quando o sequenciamento for logisticamente impraticável ou substantivamente irrelevante. Algumas vezes, no entanto, a própria questão de pesquisa exige que os diferentes tipos de dados sejam coletados simultaneamente. Por exemplo, em uma pesquisa sobre discriminação racial, o pesquisador pode investigar em que medida dimensões latentes de ideologia extraídas via análise fatorial utilizando dados de survey se conformam com o discurso dos participantes em um grupo focal. Morse $(1991$; 2003) e Smith (2008) discutem as vantagens e desvantagens de cada perspectiva.

Esquematicamente tem-se o seguinte:

Sequential explanatory strategy - Quantitativo precede o qualitativo. A análise qualitativa será realizada a partir dos resultados preliminares produzidos via análise quantitativa. Morse (1991) afirma que essa abordagem é particularmente apropriada quando o pesquisador observa resultados inesperados ou casos desviantes (outliers). O pes- 
quisador pode selecionar apenas os casos destoantes e examiná-los através de técnicas qualitativas. O exemplo típico dessa abordagem é a utilização de entrevistas (quali) para aprofundar os achados de uma pesquisa de survey (quanti).

Sequential exploratory strategy - Qualitativo precede o quantitativo. O desenho de pesquisa pode ser ou não teoricamente orientado. Morgan (1997) defende que essa abordagem também pode ser utilizada para testar hipóteses e fazer generalizações para outras amostras. Por sua vez, Creswell (2012) afirma que esse tipo de abordagem é especialmente adequado para desenvolver novos instrumentos de coleta e/ou aperfeiçoar instrumentos já existentes. O exemplo típico dessa abordagem é a utilização de grupo focal (quali) para informar o processo de elaboração do questionário (quanti).

Sequential transformative strategy - aquele procedimento em que o pesquisador utiliza o enfoque teórico como uma perspectiva ampla, cujo projeto apresenta dados quantitativos e qualitativos. Essa estratégia assegura uma estrutura de métodos para coleta de dados e para os resultados. Dentro dessa abordagem podemos desenvolver um método de coleta de dados que envolva uma abordagem sequencial ou concomitante.

Outra dimensão importante da combinação de métodos é o nível de análise em que os dados são coletados. Para Lieberman (2005), o aninhamento diz respeito ao grau em que diferentes tipos de dados são coletados para os mesmos atores, organizações ou entidades. Ou seja, em que medida os dados coletados estão delimitados a um tipo de observação que pertence a um nível de análise específica. O exemplo clássico de dados aninhados são as informações referentes ao desempenho de alunos. A unidade mais básica de observação é o desempenho do estudante, mas que por sua vez está aninhado em uma determinada turma, de uma série 
específica e dentro de uma determinada escola. A escola por sua vez integra um município que também está aninhado em um estado. Os modelos hierárquicos, também chamados de multiníveis, são a ferramenta mais adequada para lidar com dados dessa natureza. No que diz respeito ao desenho de pesquisa aninhado na perspectiva de combinação de métodos, o procedimento típico é aplicar questionários e, posteriormente, selecionar alguns respondentes a partir de critérios substantivos ou aleatoriamente para participar de entrevistas em profundidade.

Para além da combinação de tipos de técnicas e dados, a literatura mais recente tem apresentando uma nova tendência: a utilização cruzada de um tipo de técnica para analisar os dados de outra natureza. Por exemplo, utilizar um modelo multivariado para explicar a variação da quantidade de termos captados por uma análise de discurso e/ou as transcrições de entrevistas em profundidade. Por exemplo, Pearce (2002) examinou o impacto da religiosidade sobre o tamanho das famílias no Nepal. O primeiro passo foi utilizar técnicas quantitativas para examinar uma amostra de cerca de 1.800 casos. Depois disso, 28 casos destoantes foram selecionados para participar de entrevistas em profundidade. Os resultados das entrevistas e os dados colhidos durante a pesquisa de campo sugeriram a estimação de um novo modelo, que considerasse como unidade de informação a família e não o indivíduo. O ajuste do segundo modelo foi melhor do que o do primeiro.

Small (2011) distingue entre crossover analysis e integrative analysis. A abordagem crossover consiste em analisar dados qualitativos a partir de alguma modelagem formal, matemática ou estatística. Para ficar menos abstrato, suponha que depois de realizar uma análise documental histórica, entrevistas em profundidade e grupos focais, o pesquisador codifique essas informações em um banco de dados para examiná-las via estatística descritiva e/ou multivariada. Um dos componentes que possibilitou o 
desenvolvimento dessa abordagem foi o avanço computacional e o surgimento de pacotes estatísticos cada vez mais amigáveis. Atualmente, é possível examinar quantitativamente informações produzidas via análise de discurso, análise documental, notas etnográficas, etc.

Por sua vez, a abordagem integrativa prevê a utilização de diferentes técnicas para analisar um mesmo banco de dados. Para Small (2011), a ideia básica é garantir a complementariedade das técnicas: utilizar as vantagens analíticas de cada perspectiva para produzir um estudo mais robusto do que seria realizado de forma unilateral (apenas quanti ou apenas quali).

\subsection{Combinando métodos quantitativos e qualitativos}

O primeiro passo para formular um desenho de pesquisa multimétodo é dominar, minimamente, algumas das técnicas quantitativas e qualitativas. Além disso, o pesquisador deve ter claro qual é a sua questão de pesquisa e quais são os objetivos do trabalho. Yin (2006) adverte que um dos principais desafios enfrentados pelos pesquisadores que combinam métodos é garantir a unidade do desenho de pesquisa. Isso porque é comum que o trabalho acabe se fragmentando em desenhos de pesquisa paralelos. Além disso, Yin (2006) afirma que para ser considerado como método misto, o desenho de pesquisa não precisa, necessariamente, combinar técnicas quantitativas e qualitativas. Um estudo que utiliza survey e desenho experimental também pode ser considerado como método misto. Similarmente, a utilização de análise documental associada a grupo focal também se encaixa sob essa definição.

APLICAÇÕEs:

Para demonstrar como um desenho de pesquisa pode combinar técnicas quantitativas e qualitativas, iremos resumir a metodologia de dois trabalhos: (1) Bizarro, Silva e Rocha (2011) e (2) Arruda (2013). Como nosso foco repousa sobre o desenho de pesquisa, não iremos discutir os resul- 
tados substantivos desses trabalhos nem tampouco eventuais imprecisões da aplicação das técnicas. Outra coisa, não faz diferença se o trabalho é classificado como sendo de Ciência Política, Antropologia ou Sociologia. Isso porque a mesma técnica de entrevista pode ser utilizada para coleta informações sobre o posicionamento ideológico dos deputados, as aspirações profissionais dos jovens ou as percepções a respeito da relação entre religião e sexualidade no contexto urbano. Em síntese, não é a disciplina que determina a técnica, mas a natureza da questão de pesquisa.

\section{Exemplo 01}

Bizarro, Silva e Rocha (2011) realizaram um estudo de caso com o objetivo de analisar o fenômeno do "poste" ${ }^{14}$. Metodologicamente, os autores combinam análise documental ${ }^{15}$, grupo focal ${ }^{16}$, dados secundários, survey $^{17}$ e estatística multivariada. As tabelas 1 e 2 sumarizam testes de correlação entre renda, escolaridade e intenção de voto.

\footnotetext{
14 "um indivíduo lançado candidato como mero destinatário nominal dos votos de outro indivíduo, ou de um pequeno grupo de indivíduos, que por uma conveniência qualquer designariam como candidato outra pessoa em vez de saírem eles próprios candidatos" (Bizarro; Silva; Rocha, 2011: 205).

15 "Durante o levantamento que antecedeu a elaboração deste texto (até outubro de 2009), identificaram-se 182 ocorrências da expressão na grande imprensa e m blogs de opinião a designar a situação descrita na apresentação, sendo que 74\% delas (135) faziam menção direta à eleição recifense" (Bizarro; Silva; Rocha, 2011: 206).

${ }^{16}$ Para uma introdução em português à técnica de grupo focal ver Lervolino e Pelicioni (2001). Para um trabalho clássico sobre o assunto ver Morgan (1997). Para leitores interessados em aprofundar seus conhecimentos ver Byers e Wilcox (1991), Carey (1995), Delli Carpini e Williams (1994), Fern (1982), Flores e Alonso (1995).

17 "Em Recife foram empregados dados de survey organizados pelo Instituto de Pesquisas Sociais, Políticas e Econômicas (Ipespe), pelo Ibope e pelo Datafolha, além dos dados oficiais disponibilizados pelo Tribunal Superior Eleitoral. Além disso, foi realizado grupo focal, em outubro de 2009, com participantes recrutados a partir de amostra não probabilística" (Bizarro; Silva; Rocha, 2011: 206).
} 
Tabela 1. Testes de correlação Gama, Spearman e Pearson para renda do eleitor recifense e intenção do voto em João da Costa antes da campanha oficial

\begin{tabular}{ccccc}
\hline Correlação & Valor & $\begin{array}{c}\text { Erro-padrão } \\
\text { assintótico }\end{array}$ & $\begin{array}{c}\text { t-valor } \\
\text { aproximado }\end{array}$ & $\begin{array}{c}\text { Significância } \\
\text { aproximada }\end{array}$ \\
\hline Gama & 0,15 & 0,08 & 1,91 & 0,06 \\
\hline Spearman & 0,06 & 0,03 & 1,81 & 0,07 \\
\hline Pearson & 0,05 & 0,03 & 1,69 & 0,09 \\
\hline $\mathbf{N}$ (casos válidos) & $\mathbf{9 8 2}$ & & & \\
\hline
\end{tabular}

Fonte: Ipespe (Barômetro de Pernambuco, quinta rodada), maio 2008. Testes mostram associação positiva, significativa a um nível de $10 \%$.

Tabela 2. Testes de correlação Gama, Spearman e Pearson para escolaridade do eleitor recifense e intenção do voto em João da Costa antes da campanha oficial

\begin{tabular}{ccccc}
\hline Correlação & Valor & $\begin{array}{c}\text { Erro-padrão } \\
\text { assintótico }\end{array}$ & $\begin{array}{c}\mathbf{t} \text { - valor } \\
\text { aproximado }\end{array}$ & $\begin{array}{c}\text { Significância } \\
\text { aproximada }\end{array}$ \\
\hline Gama & 0,11 & 0,07 & 1,48 & 0,14 \\
\hline Spearman & 0,05 & 0,03 & 1,48 & 0,14 \\
\hline Pearson & 0,05 & 0,03 & 1,62 & 0,11 \\
\hline $\mathbf{N}$ (casos válidos) & $\mathbf{9 8 2}$ & & & \\
\hline \hline
\end{tabular}

Fonte: Ipespe (Barômetro de Pernambuco, quinta rodada), maio 2008. Testes mostram associação positiva e significativa a um nível de $15 \%$.

Por sua vez, a tabela 3 sumariza as principais motivações que explicam a preferência dos eleitores pelos candidatos a prefeito do Recife. 
Tabela 3. Principal fator para que um candidato a prefeito obtivesse o voto do eleitor recifense (agosto de 2008)

\begin{tabular}{lcc}
\hline $\begin{array}{l}\text { O que é importante para que o candidato a prefeito tenha o } \\
\text { seu voto? }\end{array}$ & Frequência & \% válido \\
\hline Não estar sendo processado pela justiça & 186 & 18,6 \\
\hline Suas propostas & 175 & 17,5 \\
\hline $\begin{array}{l}\text { Qualidades pessoais do candidato (preparo intelectual e } \\
\text { experiência profissional) }\end{array}$ & 148 & 14,8 \\
\hline Experiência do candidato em cargos políticos & 144 & 14,4 \\
\hline Oferecer ajuda pessoal & 103 & 10,3 \\
\hline Seu partido & 94 & 9,4 \\
\hline Seu histórico & 80 & 8,0 \\
\hline O apoio de outros líderes & $\mathbf{N}=1.001$ & $\mathbf{1 0 0}$ \\
\hline Nenhuma & 13 & 1,3 \\
\hline Não sabe/não respondeu & 24 & 2,4 \\
\hline \hline
\end{tabular}

Fonte: Ipespe (Barômetro de Pernambuco, quinta rodada), maio 2008.

Para além da análise quantitativa, o desenho de pesquisa também contou com grupos focais. Vejamos alguns exemplos:

Deixa para lá [risos], não faz mal não, que é que tem, né? [...] às vezes é uma besteirinha de nada, assim, um probleminha besta...Às vezes o sujeito tá com um probleminhano 
Serasa, tá devendo uma conta de luz, um carnê, e tudo, mas é gente boa [homem, $R P A^{18}$ 6, 46 anos, desempregado]

É, é sério o problema, essa estória aí...com bronca na Justiça, não pode votar no cara, não, e a turma vota mesmo porque não "se liga" nas coisas [homem, RPA 1, 22 anos, comerciário]

[...] no fim parece disputa do Sport com Santa Cruz ${ }^{19}$, e ninguém para pra pensar direito, nem quer saber quem tá certo ou tá errado...só quer mesmo que seu time ganhe e pronto. E quem perde mesmo é a cidade [homem, RPA 5, 36 anos, bancário]

Um elemento central da análise dos fragmentos do grupo focal é a contextualização das informações. Por exemplo, o leitor pode não saber o que são as RPAs, bem como prescindir de conhecimentos futebolísticos. Como regra, os autores do trabalho devem fornecer informações específicas que ajudem o leitor a conferir significado substantivo às falas dos participantes. Outro elemento desejável é confrontar os resultados observados via análise qualitativa com aqueles obtidos via técnicas quantitativas. Bizarro, Silva e Rocha (2011) fazem isso ao relatarem que:

A tabela 3 revela importante característica do eleitor recifense, mostrando que sua "racionalidade decisória" está eivada de incoerências. Em agosto de 2008, os eleitores recifenses disseram que "não estar sendo processado pela Justiça" era o principal fator para decidir o seu voto para prefeito. Pouco mais de um mês depois, o candidato João

\footnotetext{
18 "Regiões político-administrativas do Recife (RPAs). Trata da caracterização geral da cidade através de suas seis RPAs, recuperando, também, a história dessa divisão, desde 1936. Além do mais, apresenta seu processo de urbanização para, em seguida, discorrer sobre os aspectos demográficos, ecológicos, de uso do solo e físico-ambientais. São seis regiões definidas: região centro (RPA 1); região Norte (RPA 2); região Nordeste (RPA 3); região Oeste (RPA 4); região Sudeste (RPA 5) e região Sul (RPA 6)" (Bizarro; Silva; Rocha, 2011: 215).

19 "Times de futebol que reúnem o maior número de torcedores na capital pernambucana" (Bizarro; Silva; Rocha,, 2011: 215).
} 
da Costa foi eleito no primeiro turno, apesar da Justiça tê-lo condenado, em primeira instância, por uso da máquina da prefeitura. Explorada tal situação no grupo focal realizado em outubro de 2009, os eleitores primeiramente disseram não haver relação entre uma coisa e outra, depois, disseram que se tratava de uma contradição do eleitorado (Bizarro; Silva; Rocha, 2011: 214).

\section{Exemplo 02}

Arruda (2013) empregou uma combinação de métodos para desvendar os fatores que orientam a decisão do voto do eleitor cuiabano. Mais precisamente, a análise incidiu sobre a eleição municipal de 2012. A estratégia metodológica definida para o trabalho tinha dois grandes desafios: (1) identificar como o eleitor cuiabano entendia o momento político e (2) constatar como ele se comportou. Para tanto, o autor combinou: análise documental ${ }^{20}$, dados secundários, grupo focal ${ }^{21}$, survey ${ }^{22}$ e estatística multivariada.

As duas primeiras técnicas (análise documental e dados secundários) foram utilizadas para contextualizar o objeto. Vale dizer, são raros os exemplos de emprego adequado dessas técnicas, embora elas sejam duas das mais utilizadas pelos pesquisadores em Ciências Sociais. De acordo

\footnotetext{
${ }^{20}$ De acordo com Arruda (2013), a análise documental consistiu no acompanhamento da cobertura jornalista sobre o pleito em alguns dos principais veículos de comunicação do Estado do Mato Grosso. Precisamente foram observadas as reportagens sobre o tema no período de $\mathrm{X}$ a Y veiculadas no MidiaNews, Rdnews e Gazeta Digital.

${ }^{21}$ De acordo com Arruda (2013), "foram realizados 13 grupos focais entre os dias 14 e 26 de outubro, sendo que cada grupo focal contou com um total de 10 participantes, sendo homens e mulheres, com idades entre 18 e 45 anos, das classes C e D" (ARRUDA, 2013: 13). Os grupos foram mediados a partir de um roteiro mínimo e tiveram todo conteúdo gravado em áudio.

${ }^{22}$ Foram utilizados dados sobre as eleições municipais anteriores disponibilizados pelo TSE. Mais precisamente, esses dados serviram principalmente para retratar o quadro das eleições municipais nos quatro pleitos anteriores, ou seja, no período 1997 - 2012. De como complementar, recorreu-se também a números de pesquisa de opinião realizados pelo IBOPE meses antes da realização do pleito (2012).
} 
com a literatura, os dados secundários devem ser utilizados na fase exploratória da pesquisa. Normalmente, com a finalidade de esclarecer a questão de pesquisa e/ou descobrir estratégias de abordagem do tema anteriormente utilizadas (Fayyad; Piatetsky-Shapiro, 1996; Berry; Linoff, 1999). Arruda (2013) utilizou a técnica com a primeira finalidade. Seu intuito foi deixar claro "os antecedentes da política cuiabana" e, dessa forma, caracterizar seu objeto de análise (eleição municipal de Cuiabá 2012). Esse objetivo fica claro nas passagens abaixo:

Analisando as eleições para prefeito em Cuiabá, a partir da primeira eleição (1985) após o período de redemocratização, podemos observar um melhor desempenho dos partidos de centro-direita, em especial do PSDB (...). Podemos observar o domínio dos partidos de centro-direita também na Câmara Municipal de Cuiabá. Novamente o PSDB se destaca ao analisarmos as legislaturas no período de 1997 a 2016. (Arruda, 2013: 45).

Já com o grupo focal e o survey, Arruda (2013) buscou cumprir o primeiro grande desafio da análise: identificar como o eleitor cuiabano entendia o momento político. De acordo com a literatura, os grupos focais fornecem elementos importantes, dentre outros, para: formulação das questões de pesquisa, elaboração de instrumentos para pesquisa experimental e quantitativa e formulação de hipóteses (Morgan, 1997; Minayo, 2000; Veiga; Gondim, 2001). Arruda (2013) segue essa orientação e utiliza os dados extraídos dos grupos focais para desenhar hipóteses e escolher a estratégia de verificação delas.

Todavia, a análise do comportamento do eleitor cuiabano é complementada pela realização de um survey. Para Converse e Presser (1986), esse instrumento se consolidou como uma das melhores ferramentas para coleta de informações. Arruda (2013) utiliza dados de survey para examinar a associação entre "as intenções de voto dos eleitores cuiabanos e um conjunto significativo de variáveis de natureza socioeconômica, política e 
emotiva" (Arruda, 2013: 13). O autor articula a análise desses dados com as falas dos membros dos grupos. Dessa forma, deixa clara sua opção por utilizar os métodos de forma complementar. Por exemplo:

O grande destaque das avaliações dos chefes do executivo ficou por conta de Chico Galindo (PTB). O ex-prefeito apresentou um alto índice de rejeição, 67\% da amostra, conforme podemos observar na tabela abaixo. (...) ste alto índice de rejeição apresentado por Galindo em nosso survey, corrobora com estas falas retiradas dos grupos focais realizados no segundo turno:

"Chico Galindo e sua poeira zero. Só sei disso que ele fez!". (P4G1)

"Pra mim é isso: poeira zero: administração zero!" (P6G1) "O povo não é mais burro, não! E o Galindo sabe. Tanto que nem se candidatou, ele não ia ganhar de jeito nenhum!" (P7G1)

"Chico Galindo! Nem me fale esse nome!" (P1G1)

"Ele não sabe administrar! Caiu de bobo na conversa de Wilson Santos e só agora tá vendo que roubada ele pegou". (P2G2)

"Antes vinha água dia sim, dia não. Depois vinha num certo horário do dia. Só de noite, quase. Como que faz pra lavar roupa de madrugada? Agora parece que tá pior!" (P8G2) (Arruda, 2013: 73-74).

Por fim, Arruda (2013) utiliza a estatística multivariada para explicar o voto do eleitor cuiabano no segundo turno. Em particular, o autor emprega um modelo de regressão logística. Os resultados sugerem que "a probabilidade de as mulheres votarem em Mauro Mendes é menor do que a dos homens. Em contrapartida a probabilidade do candidato do PSB ser votado é maior entre aqueles com maior escolaridade" (Arruda, 2013: 79).

Em resumo, apoiado numa estratégia que combina métodos qualitativos e quantitativos, Arruda (2013) foi capaz de apontar os parâmetros da decisão do voto do eleitor cuiabano, concluindo que: 
Os resultados obtidos em nossa pesquisa, nos levam a uma junção de fatores para a explicação do voto do eleitor cuiabano nessa eleição de 2012. Tais fatores estão abarcados pela união das teorias do comportamento eleitoral existentes, porém destacamos uma nova corrente, defendida por Lavareda (2011), que busca desvendar o voto por meio de variáveis emotivas. Em nossa análise, como base nos dados apresentados em nosso modelo de regressão binomial e nos grupos focais, podemos observar um eleitor emotivo (Arruda, 2013: 86).

\section{Considerações Finais}

Ninguém pode ter tudo. Cada opção metodológica tem vantagens e limitações. Por exemplo, muitas vezes os desenhos experimentais não apresentam os mecanismos subjacentes que explicam como as variáveis independentes influenciam a variável dependente. Muitas vezes não é possível saber em que medida o caso escolhido representa um outlier ou uma observação típica de uma determinada distribuição. Em geral, pesquisas com $\mathrm{N}$ grande (large $N$ studies) não apresentam informações suficientes para entender o contexto específico das unidades observadas, assim como é muito difícil garantir a replicabilidade em entrevistas e observação participante. É exatamente contra essas limitações que repousa o ganho analítico da abordagem multimétodo.

Quais são as vantagens da triangulação de métodos e técnicas de pesquisa? Essa foi a questão de pesquisa inicial deste trabalho. Apesar de ser consensual a importância da combinação de métodos quantitativos e qualitativos, ainda são raros os trabalhos que efetivamente integram diferentes técnicas de pesquisa. Este artigo apresentou uma introdução aos métodos mistos com o objetivo de difundir a abordagem multimétodo nas Ciências Sociais brasileira. Em termos substantivos, nossa meta 
é fornecer um ponto de partida para que pesquisadores interessados no assunto possam elaborar desenhos de pesquisas combinando técnicas quantitativas e qualitativas com o objetivo de produzir inferências mais robustas sobre a realidade.

Ranulfo Paranhos é Doutor e Mestre em Ciência Política pela Universidade Federal de Pernambuco (DCP/UFPE). Professor Adjunto do Instituto de Ciências Sociais da Universidade Federal de Alagoas (ICS/UFAL) e professor do Curso de Especialização em Educação em Direitos Humanos - EADHESP (UFAL).

$\sum$ ranulfo.al@hotmail.com

Dalson Britto Figueiredo Filho: é Doutor em Ciência Política pela Universidade Federal de Pernambuco (UFPE, 2012). Professor Adjunto do Departamento de Ciência Política, vice-coordenador do Programa de Pós-graduação em Ciência Política (PPGCP - UFPE). $\$ dalsonbritto@yahoo.com.br

Enivaldo Carvalho da Rocha é Doutor em Engenharia de Produção pela COPPE da Universidade Federal do Rio de Janeiro. Atualmente é professor titular da Universidade Federal de Pernambuco, Vice-diretor do Centro de Filosofia e Ciências Humanas (CFCH). $\$ enivaldocrocha@gmail.com

José Alexandre da Silva Júnior é Doutor em Ciência Política pela Universidade Federal de Pernambuco (2013). Professor do Instituto de Ciências Sociais (ICS) da Universidade Federal de Alagoas e do Mestrado Profissional em Políticas Públicas da Universidade Federal de Pernambuco (UFPE). $>$ jasjunior2007@yahoo.com.br

Diego Freitas é Doutor em Ciência Política pela Universidade Federal de São Carlos. Professor do Programa de Pós-Graduação em Sociedade, Tecnologias e Políticas Públicas do Centro Universitário Tiradentes e Professor Colaborador do Programa de Pós-Graduação em Saúde e Ambiente da Universidade Tiradentes. $\triangle$ diegofreitasrodrigues@outlook.com 


\section{Referências}

1. ARRUDA, M. V. T. Como vota o eleitor cuiabano? Evidências das eleições municipais, dissertação de mestrado defendida pelo programa de pós-graduação em Ciência Política da Universidade Federal de Pernambuco (UFPE), 2013.

2. BERRY, M. J. A.; LINOFF, G. Data mining techniques - for marketing, sales, and customer support. New York, John Wiley \& Sons, 1999.

3. BIZARRO, A. M.; SILVA, A. L da; ROCHA, E. C. "Luzes do 'poste': análise da eleição para prefeito do Recife em 2008". In: LAVAREDA, A.; TELLES, H. (Orgs.). Como o eleitor escolhe seu prefeito. Campanha e voto nas eleições municipais. Rio de Janeiro: Editora FGV, 2011.

4. BRADY, H.; COLLIER, D. Rethinking social inquiry: Diverse tools, shared standards. Lanham, MD: Rowman \& Littlefield, 2004.

5. BRYMAN, A. The Debate about Quantitative and Qualitative Research: A Question of Method or Epistemology? The British Journal of Sociology vol. 35, no. 1 (Mar., 1984), pp. 75-92.

6. BRYMAN, A.; BELL, E. Business research methods (2nd ed). Oxford: Oxford University Press, 2006.

7. BYERS, P.; WILCOX, J. R. Focus group. A qualitative opportunity for researchers. Journal of Business Comunication, 28(1), 67-78, 1991.

8. CARACELLI, V. J., \& GREENE, J. C. "Crafting mixed-method evaluation designs" In: GREENE, J. C.; CARACELLI, V. J. (Eds.). Advances in mixed-method evaluation: The challenges and benefits of integrating diverse paradigms. San Francisco: Jossey-Bass, 1997.

9. CAREY, M. A. Issues and appplication of focus group. Qualitative Health Research, 5(4), 414-420, 1995.

10. CASTRO, F. G. et al. A Methodology for conducting integrative mixed methods research and data analyses. Journal of Mixed Methods Research, v. 4, n. 4, p. 342-360, 2010.

11. CONVERSE, J. M.; PRESSER, S. Survey Questions: Handcrafting the Standardized Questionnaire. Sage University Paper Series on Quantitative Applications in the Social Sciences, 07-063. Thousand Oaks, California. Sage Publications. 80 p., 1986.

12. CRESWELL, J. W. Research design: qualitative, quantitative, and mixed methods approaches. Thousand Oaks, California: Sage, 2009.

13. CRESWELL, J. W.; PLANO CLARK, V. L. Designing and conducting mixed methods research. 2nd. Los Angeles: SAGE Publications, 2011. 
14. CRESWELL, J. W. Qualitative Inquiry and Research Design: Choosing Among Five Approaches. Thousand Oaks, CA: Sage, 2012.

15. DAHL, R. A. Poliarquia: participação e oposição. 1a ed. - São Paulo: EDUSP, 2012.

16. DELLA PORTA, D.; KEATING, M. (eds). Approaches and Methodologies in the Social Sciences. A Pluralist Perspective, Cambridge: Cambridge University Press, 2008.

17. DELLI CARPINI, M. X.; WILLIAMS, B. "Fictional" and "Non-Fictional" Television Celebrates Earthday. Cultural Studies, 8:74-98, 1994.

18. DENZIN, N. K. The values of social sciences. Nueva York: Aldine, 1970.

19. FAYYAD, U.; PIATETSKY-SHAPIRO, G. From data mining to knowledge discovery in databases. Al Magazine, 17, 37-54, 1996.

20. FERN, E. F. The use of focus group for idea generation: the effects of group size, group type, acquaintanceship, and the moderator on response quantity and quality. Journal of Marketing Research, Vol, 19, Winter, 1982.

21. FLORES, J. G.; ALONSO, G. C. Using focus groups in educational research: Exploring teachers' perspectives on educational change. Evaluation Review 19, 84- 101, 1995.

22. GORARD, S.; TAYLOR, C. Combining methods in educational and social research, London: Open University Press, 2004.

23. JOHNSON, R. B.; ONWUEGBUZIE; A. J. Mixed Methods Research: A Research Paradigm Whose Time Has Come, Educational Researcher, vol.33, 2004, pp.14-26, 2004.

24. JOHNSON, R. B.; ONWUEGBUZIE, A. J.; TURNER, L. A. Toward a Deinition of Mixed Methods Research. Journal of Mixed Methods Research, vol.1, pp.112-133, 2007.

25. JICK, T. D. Mixing qualitative and quantitative methods: Triangulation in action. Administrative Science Quarterly, 24, 602-611, 1979.

26. KALTON, G. Introduction to survey sampley. Beverly Hills, Sage Puplicaations, 1983.

27. KING, G., KEOHANE, R.; VERBA, S. Designing Social Inquiry: Scientific Inference in Qualitative Research. Princeton. N.J.: Princeton University Press, 1994.

28. LERVOLINO, S. A.; PELICIONI, M. C. F. A utilização do grupo focal como metodologia qualitativa na promoção da saúde. Rev. Esc. Enf., USP, 35 (2):11521, jun 2001. 
29. LIEBERMAN, E. Nested Analysis as a Mixed-Method Strategy for Comparative Research. American Political Science Review, 99, 3: 435-452, 2005.

30. MARQUES, E. C. L. et al. Dossiê: métodos e explicações da política. Rev. Bras. Ciências. Soc., São Paulo, v.22, n.64, jun. 2007.

31. MARSH, D.; FURLONG P. "A Skin not a Sweater: Ontology and Epistemology in Political Science", In: D. Marsh; G. Stoker (eds.), Theory and Methods in Political Science, Basingstoke: Palgrave, p. 17-44, 2002.

32. MINAYO, M. C. S. O desafio do conhecimento: pesquisa qualitativa em saúde. 7. ed. São Paulo: Hucitec, 2000.

33. MORGAN, D. L. Focus group as qualitative research. London: Sage, 1997.

34. MORGAN, D. Paradigms Lost and Pragmatism Regained, Methodological Implications of Combining Qualitative and Quantitative Methods. Journal of Mixed Methods Research January 2007 vol. 1 no. 1 48-76

35. MORSE, J.M. Approaches to qualitative-quantitative methodological triangulation. Nursing Research, 40(1), 1991, p.120-132.

36. MORSE, J.M. Procedures and Practice of Mixed Method Design: Maintaining Control, Rigor, and Complexity In: TASHAKKORI, A.; TEDDLIE, C. (Eds). The Sage Handbook of Mixed Methods Research in Social \& Behavioral Research, London, Sage, 2003, pp.189-208

37. NIGLAS, K. The combined use of qualitative and quantitative methods in educational research. Tallinn Pedagogical University, 2004. Dissertations on Social Sciences. Acesso em 20, out 2014, http://www.tlulib.ee/files/arts/95/ nigla32417030233e06e8e5d471ec0aaa32e9.pdf.

38. PEARCE, L. D. Integrating survey and ethnographic methods for systematic anomalous case analysis. Sociological Methodology, 32, 103-132, 2002.

39. PFEFFERMANN, D.; RAO, C. R. (Eds.). Handbook of Statistics. Volume 29A, Sample Surveys: Design, Methods and Application and Volume 29B, Sample Surveys: Inference and Analysis. New York: Elsevier, 2009.

40. RUIZ, F. M. Pesquisa qualitativa e pesquisa quantitativa: complementariedade cada vez mais enriquecedora. Administração de Empresas em Revista, Curitiba, n. 3, p. $37-47,2004$.

41. SANDELOWSKI, M. Focus on research methods combining qualitative and quantitative sampling, data collection, and analysis techniques in mixed-method studies. Research in Nursing \& Health, v. 23, n.3, p. 246-255, June 2000. 
42. SMALL, M. L. How to conduct a mixed methods study: Recent trends in rapidly growing literature. Annual Review Sociology, 37:57-86, 2011

43. SMITH, J.A. Qualitative Psychology - A Practical Guide to Research Methods, 2nd ed., London: Sage, 2008.

44. TARROW, S. Bridging the Quantitative-Qualitative Divide in Political Science. American Political Science Review, vol.89, vol.2, pp.471-74, 1995.

45. TASHAKKORI, A.; TEDDLIE, C. Mixed methodology: combining qualitative and quantitative approaches. Thousand Oaks, Calif.: Sage, 1998.

46. TASHAKKORI, A.; TEDDLIE, C.(Eds.) The Sage Handbook of Mixed Methods Research in Social \& Behavioral Research, London, Sage, 2003.

47. TILLY, C. Mechanisms in political process. Annual Review of Political Science, 4, 21-41, 2001.

48. VEIGA, L.; GONDIM, S. M. G. A utilização de métodos qualitativos na Ciência Política e no Marketing Político. Opin. Publica, Campinas, v. 7, n. 1, p. 1-15, 2001.

49. WOOLLEY, G. The assessment of reading comprehension difficulties for reading intervention. Australian Journal of Learning Difficulties, 13(1), 51-62, 2008.

50. YIN, R. K. Estudo de caso: planejamento e métodos. 3 ed. Porto Alegre: Bookman, 2006.

Recebido em: 17/03/2015

Aceite Final: 13/07/2015 